\title{
Wavelet analysis of a large sample of AGN at high radio frequencies ${ }^{\star}$
}

\author{
T. Hovatta ${ }^{1}$, H. J. Lehto ${ }^{2,3}$, and M. Tornikoski ${ }^{1}$ \\ 1 Metsähovi Radio Observatory, TKK, Helsinki University of Technology, Metsähovintie 114, 02540 Kylmälä, Finland \\ e-mail: tho@kurp.hut.fi \\ 2 Tuorla Observatory, University of Turku, Väisäläntie 20, 21500 Piikkiö, Finland \\ 3 Department of Physics, 20140 University of Turku, Finland
}

Received 15 May 2008 / Accepted 18 June 2008

\begin{abstract}
Aims. We have studied the characteristic timescales of 80 AGNs at 22, 37 and $90 \mathrm{GHz}$ examining the properties of the wavelet method and comparing them to traditional Fourier-based methods commonly used in astronomy.

Methods. We used the continuous wavelet transform with the Morlet wavelet to study the characteristic timescales. We also gain information when the timescale is present in the flux curve and if it is persistent or not.

Results. Our results show that the sources are not periodic and changes in the timescales over a long time are common. The property of wavelets to be able to distinguish when the timescale has been present is superior to the Fourier-based methods. Therefore we consider it appropriate to use wavelets when the quasi-periodicities in AGNs are studied.
\end{abstract}

Key words. galaxies: active - methods: statistical - radio continuum: galaxies

\section{Introduction}

Wavelet methods have been used for the past 10 years to study the variability timescales in different types of astronomical objects (e.g. Foster 1996; Priestley 1997; Scargle 1997). Wavelets have properties superior to Fourier-based methods, the most useful being the locality of the timescale. In Fourier-based methods a single sinusoid is fitted to the whole light curve, whereas wavelets enable us to study temporally local timescales or periods that are present in the light curves. This is a very good property when studying flux curves of AGNs, which are almost never strictly periodic over long times.

Wavelets were used to study variability timescales of a larger sample of AGNs by Kelly et al. (2003). They used the cross-wavelet transform to study quasi-periodic variations in the Pearson-Readhead VLBI sample, monitored by the University of Michigan Radio Observatory (UMRAO). A quasi-periodic behaviour was found in a little over half of the 30 sources they studied. Individual sources have also been studied for quasiperiodicity and periodicity using wavelets (e.g. Hughes et al. 1998; Lehto 1999; Kadler et al. 2006; Ciprini et al. 2007).

In Hovatta et al. (2007, hereafter Paper I), we studied the variability timescales of a large sample of AGNs using the structure function (SF), the discrete autocorrelation function (DCF) and the Lomb-Scargle periodogram. We found that larger flares in these sources happen quite rarely, only once in 4-6 years while short-term variations are continuously present in the flux curves. Also, many of the sources had changed their behaviour during the 25 years of monitoring which is one reason why we wanted to study the sample again using wavelets. In Paper I we also studied the properties of the methods and their differences

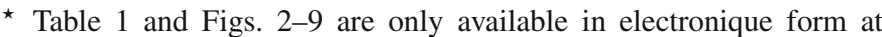
http://www. aanda.org by searching for the characteristic timescales. We noticed that there are several problems when using periodograms to study the timescales because none of the sources in our sample showed strict periodicities in their flux curves or were well characterised by sinusoids.

In this paper we will compare the results from this wavelet analysis to Paper I. This paper is organised as follows: in Sect. 2 we describe the source sample and data used. Section 3 includes the description of the method and in Sect. 4 we present the results. The discussion is in Sect. 5 and the conclusions are drawn in Sect. 6.

\section{The sample and observations}

We used the same sample of 80 AGNs selected from the Metsähovi monitoring list that was used in Paper I. The sample consists of $24 \mathrm{BL}$ Lacertae Objects (BLOs), 23 Highly Polarised Quasars (HPQs), 28 Low Polarisation Quasars (LPQs) and 5 Radio Galaxies (GALs). The five sources with no information about their optical polarisation are considered to be LPQs in this study and are included in the numbers above.

All sources have been monitored for at least 10 years at 22 or $37 \mathrm{GHz}$. These are all bright sources with a flux density of at least $1 \mathrm{Jy}$ in the active state in at least one of the two frequencies. We used the 22, 37 and $87 \mathrm{GHz}$ data obtained with the Metsähovi 14-metre radio telescope over 25 years of monitoring (Salonen et al. 1987; Teräsranta et al. 1992, 1998, 2004, 2005; Nieppola et al. 2007). The $37 \mathrm{GHz}$ data of HPQs, LPQs and GALs from December 2001 until March 2005 are partially unpublished. The details of the observation method and data reduction processes are given in Teräsranta et al. (1998). In addition we used $90 \mathrm{GHz}$ data obtained with the Swedish-ESO Submillimetre Telescope (SEST) at La Silla, Chile, which was used in our monitoring campaign to sample the high frequency, 
90 and $230 \mathrm{GHz}$ variability of southern and equatorial sources (Tornikoski et al. 1996). The monitoring campaign at SEST lasted from 1987 to 2003 from which the data from 1994.5-2003 are unpublished. Complementary high frequency data at $90 \mathrm{GHz}$ were also collected from the literature (Steppe et al. 1988, 1992, 1993; Reuter et al. 1997). The median intervals between the observations at 22,37 and $90 \mathrm{GHz}$ are 31,41 and 47 days, respectively. The values depend on the source and at $37 \mathrm{GHz}$ the minimum average sampling rate was 6.8 days for the source $3 \mathrm{C} 84$ which is used as a secondary calibrator in the Metsähovi observations. The maximum average value at $37 \mathrm{GHz}$ was 186.4 days for the source $2234+282$.

\section{The wavelet method}

We have analysed the light curves using Morlet wavelets. They can be understood as local wave packets. The method is closely related to chirp analysis methods, Short Time Fourier Transform methods, Gabor transforms and also to the sonogram or spectrogram analysis used widely in audio signal processing. In wavelet analysis the length of the analysis "package", the kernel, is proportional to the timescale of interest, or in terms of frequency, proportional to the inverse of the frequency. We have used a Morlet wavelet, which can be understood mathematically as a localised plane wave tapered by a Gaussian function. For longer timescales of interest we thus use lower frequency sinusoids than for higher frequencies according to the following equations. Wavelet transforms are functions of both frequency, $f$, (or the respective timescale) and the local time, $\tau$.

$g^{*}(f, \tau)=\exp \left(-\mathrm{i} c f(t-\tau)-\frac{1}{2}(f(t-\tau))^{2}\right)$,

where we adopt $c=2 \pi$, which characterises the amount of tapering. The power of the wavelet transform, known as the scalogram, is defined for evenly spaced data as

$W(f, \tau)=f \cdot\left(S^{2}(f, \tau)+C^{2}(f, \tau)\right)$,

where

$$
\begin{aligned}
& S(f, \tau)=\sum_{j=1}^{N} m_{j} \sin \left(2 \pi f\left(t_{j}-\tau\right)\right) \exp \left(\frac{-1}{2}\left(f\left(t_{j}-\tau\right)\right)^{2}\right), \\
& C(f, \tau)=\sum_{j=1}^{N} m_{j} \cos \left(2 \pi f\left(t_{j}-\tau\right)\right) \exp \left(\frac{-1}{2}\left(f\left(t_{j}-\tau\right)\right)^{2}\right),
\end{aligned}
$$

where $m_{j}$ and $t_{j}$ are respectively the flux density and the time of the observation $j$.

For unevenly spaced data we have to weight each point with the inverse of the local density of points. The locality, a property of wavelets and other similar methods mentioned above, is in strong contrast to global methods such as Fourier transforms or structure functions, which treat the whole light curve as one entity. A change in a few critical points can change the final result in global methods rather significantly, whereas in wavelet analysis and other local methods a chance point in the data will only affect the analysis locally. As we gain information about the local nature of the signal we lose in the resolution with which we can determine the timescale (or frequency) of the signal. The length of the kernel used limits our resolution to about 0.07 in log space, which is significantly worse than what would be obtained e.g. from the Fourier analysis of a long lasting sinusoid with a constant period. Wavelet transforms with longer kernels could also be applied to the data as intermediate forms approaching Fourier transforms in accuracy, but in doing so one loses the locality information according to

$\Delta t \Delta v>\delta$

which is analogous to the Heisenberg uncertainty principle. Here $\delta$ can be considered as unity if we are interested in separating two nearby peaks. If we rather wish to measure the location of a single signal in weakly noisy data then $\delta$ is of the order of $\sqrt{(1 / N)}$, where $N$ is the local number of points.

First we calculate the scalogram for our unevenly spaced data. We then calculate a scalogram of normally distributed Gaussian white noise with a variance of unity sampled as was the original data. By repeating this 20 times we get a good estimate of the scalogram of this white noise. We then normalise our original scalogram by the white noise scalogram. If our data were pure noise, this noise-normalised scalogram would have everywhere an expected value equal to the variance of our measurements. Alternatively, we argue that by taking the square root of the noise-normalised scalogram and dividing it by the (known) measurement errors we get an estimate of the significance of the variations detected. If a variability timescale has a variance of $>9$ as expected from measurement errors, we consider that a significant detection and if the variance is between 4 and 9 times the variance of measurements, we consider that a marginal case (marked as a weak timescale in Table 1). These numbers were selected to represent " 2 and 3 sigma" levels, but we caution that these should be considered indicative only, as we have not yet carried out full simulations of the probability density distributions of the noise properties in noise-normalised scalograms.

\section{Wavelet timescales}

\subsection{Interpretation}

The advantage of wavelets is that in addition to finding the variability timescale in the frequency domain, we can see at what moments it has been present. This enables us to see whether the timescale is present during the whole observed time or is transient. In addition we can identify typical flare timescales i.e. flare duration or rise and decay times and notice whether one large outburst dominates the timescale obtained. Also short-term variability is easily detected.

We searched for both long-term variability, which is present most of the time in the flux curves, and typical timescales in which the flares occur. For the long-term timescales we first identified the timescale from the frequency axis and then determined how long it has been present on the time axis. This way we can study how many times the timescale has repeated during the time it has been present and also how long time it has been present compared to the total observing period. Figure 1 shows the wavelet transform for the source $1156+295$ (4C 29.45). We find a timescale of 3.4 years $\left(10^{0.53}\right.$ years $)$ which is present in the latter half of the flux curve, starting at 1995. The timescale is marked with a circle in the plot. Furthermore we identified typical flare timescales in the cases where more than one flare occurs on approximately the same timescale. For $1156+295$ it is seen at 1.7 years $\left(10^{0.23}\right.$ years $)$. This timescale is also shown in the plot as circles at the beginning of the time series at the time of the first two flares and later simultaneously with the long-term trend. The timescale can be identified with the durations of these flares which are of about 1-2 years. 


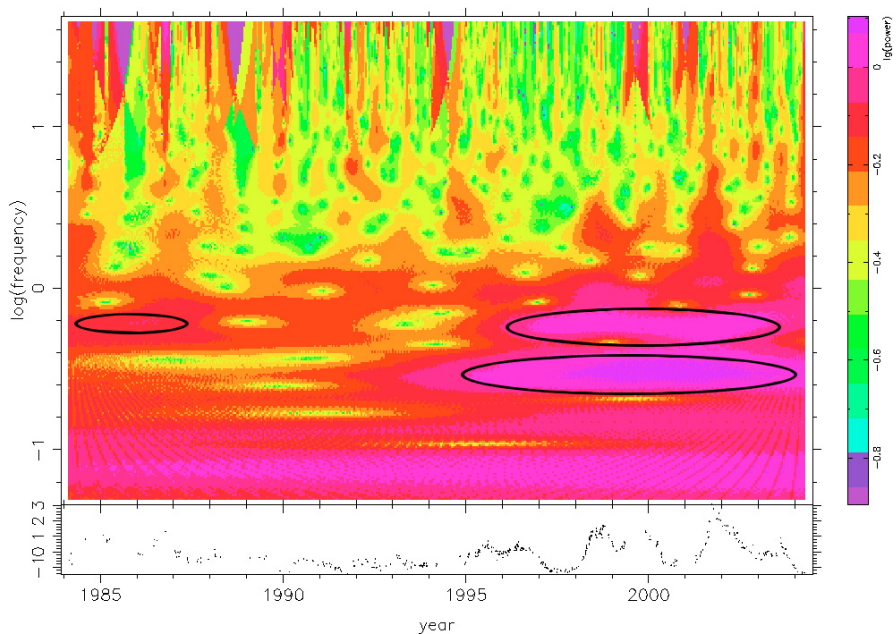

Fig. 1. Wavelet transform of the source $1156+295$ at $22 \mathrm{GHz}$. The $y$-axis is frequency 1 /year so that a timescale of 1 year corresponds to frequency 0 and a timescale of 10 years corresponds to frequency -1 in the log-axis. A long-term variability timescale of 3.4 years $\left(10^{0.53}\right.$ years $)$ is visible in the latter half of the flux curve starting at 1995. A flare timescale of 1.7 years $\left(10^{0.23}\right.$ years $)$ is also seen.

Often the long-term timescales are either slowly shortening or increasing in time which is seen as rising or declining trends in the wavelet plot. These are often connected to flares and usually the timescales shorten towards the peak of a large flare.

\subsection{Long-term trends}

We were able to determine a long-term timescale for 122 cases out of the total 192 cases for which we calculated the wavelet transform. In addition to the timescale, we determined how long it is present in the flux curve. This way we were able to calculate how many times the cycle has repeated itself. The timescales are listed in Table 1 (only available in electronic form), where the B1950-name, a well-known alias (when applicable), classification, total monitoring time, timescale and number of cycles and timescale of flares are tabulated for each frequency band. If there is more than one timescale present the most significant one is given first. In 18 sources ( $\sim 1 / 4$ of the sample) we found long-lasting cycles, continuing for at least four cycles. Five of these sources showed cycles at two separate frequencies and three sources at all the three frequency bands. There were 31 cases where the variability timescale did not last for two cycles. In Table 2 we have calculated averages and medians for the timescales continuing for at least two cycles. They are given for each frequency and source class separately. In addition we corrected the timescales for redshift and these averages are also shown in Table 2.

We ran a Kruskal-Wallis analysis to study whether the timescales at different frequencies and source classes differ from each other. The timescales at $90 \mathrm{GHz}$ differ from lower frequencies with a $95 \%$ confidence limit with the timescales being shorter. The result can be affected by the smaller number of sources at $90 \mathrm{GHz}$ and shorter monitoring period which also shortens the timescales obtained at $90 \mathrm{GHz}$. We studied the differences between the source classes at 22 and $37 \mathrm{GHz}$ and at both frequencies the classes do not differ significantly from each other if the observational uncorrected timescales are considered. When we study the redshift corrected timescales we see significant differences between the BLOs and LPQs at $37 \mathrm{GHz}$ and in addition there is strong indication that also the HPQs and BLOs differ. Similar results were obtained in Paper I, when periodogram timescales were studied.

We were also interested in sources in which the timescale is present during most of the flux curve. By visually extracting the duration of the timescale from the wavelet plot, we calculated how much of the total monitoring time it has been present. In 27 cases the timescale was present at least $90 \%$ of the total observing time. Seven of these, however, were cases in which the timescale had not repeated twice during the period it was visible.

\subsection{Flare timescales}

In addition to long-term trends we determined a flare timescale which describes the duration or rise and decay times of flares. These timescales are seen in the wavelet transform at the times of the outbursts. We determined such a timescale in 53 cases. They are also tabulated in Table 1. We have taken into account cases for which a similar timescale seems to be present during more than one flare and therefore represented a typical flare timescale for the source. The values we obtained vary between $0.34-2.17$ years at 22 and $37 \mathrm{GHz}$. The average value at $22 \mathrm{GHz}$ is 1.16 years and at $37 \mathrm{GHz}$ it is 1.03 years. At $90 \mathrm{GHz}$, there were only 6 sources which showed repeating flares and the timescales were shorter, varying between 0.34 and 0.87 years, with an average of 0.69 years.

\subsection{Individual sources}

Even though none of the sources studied here seems to show a strict periodicity in radio frequencies, many of them show quasiperiodic behaviour. We are interested in sources that have a variability timescale lasting four cycles in at least two of the frequency bands. There are eight such sources and their properties are discussed in more detail below. The wavelet plots for these sources at $37 \mathrm{GHz}$ are shown in Figs. 2-9 (only available in electronic form).

DA $55(0133+476)$ : This HPQ source has been monitored in Metsähovi for over 20 years at 22 and $37 \mathrm{GHz}$ and for almost 15 years at $90 \mathrm{GHz}$. It has shown high apparent pattern velocity of $18 c$ in the jet (Piner et al. 2007) and has been also a target of many other studies using Very Long Baseline Interferometry (VLBI) observations (e.g. Lister 2001; Kellermann et al. 2004; Lister \& Homan 2005). It is also included in the wavelet study of Kelly et al. (2003) which uses the UMRAO 4.8, 8, $14.5 \mathrm{GHz}$ monitoring data. They found a timescale of 2.3 years for the source at $14.5 \mathrm{GHz}$. This is very close to the timescale of 2.2 years which we obtained for the source at both 22 and $37 \mathrm{GHz}$. At $90 \mathrm{GHz}$ we only found a flare timescale of 0.7 years for the source. The long-term timescale in our analysis has a rising trend so that it is shortening towards a peak of a large flare. The timescale is present only for about $40 \%$ of the flux curve at both 22 and $37 \mathrm{GHz}$ but still has repeated 4.4 times at both of the frequency bands. In Paper I we could not find any DCF or periodogram timescale for the source. When the flux curve is examined we can clearly see that the timescale obtained here is related to a period when the source had many similar flares and the total flux level rises. This also explains the rising trend in the wavelet plot which is caused by more frequent individual flares towards the peak of the larger event.

$3 C 120(0430+052)$ : This radio galaxy has been monitored in Metsähovi for over 20 years at 22 and $37 \mathrm{GHz}$ and for 15 years at $90 \mathrm{GHz}$. It is a well-studied object at all wavelengths and has 
Table 2. Averages and medians of long-term timescales that have lasted for at least two cycles. Values are given for each frequency and source class separately. Also the redshift corrected averages are shown.

\begin{tabular}{llllllllll}
\hline \hline Freq & Type & ALL & $\mathrm{N}$ & BLO & $\mathrm{N}$ & HPQ & $\mathrm{N}$ & LPQ & $\mathrm{N}$ \\
\hline 22 & average & 4.6 & 37 & 5.1 & 13 & 4.8 & 10 & 4.2 & 10 \\
& redshift corr. & 3.0 & 37 & 3.6 & 13 & 2.7 & 10 & 2.4 & 10 \\
& median & 4.3 & 37 & 5.5 & 13 & 4.9 & 10 & 3.9 & 10 \\
\hline 37 & average & 4.4 & 41 & 5.2 & 10 & 4.5 & 15 & 3.8 & 12 \\
& redshift corr. & 2.8 & 40 & 4.2 & 9 & 2.4 & 15 & 2.1 & 12 \\
& median & 4.3 & 41 & 4.5 & 10 & 4.3 & 15 & 3.7 & 12 \\
\hline 90 & average & 2.9 & 11 & 2.6 & 4 & 3.0 & 4 & 3.5 & 2 \\
& redshift corr. & 1.9 & 11 & 1.6 & 4 & 1.9 & 4 & 2.3 & 2 \\
& median & 2.7 & 11 & 2.3 & 4 & 3.1 & 4 & 3.5 & 2 \\
\hline
\end{tabular}

been a target of many multiwavelength campaigns. Its radio jet has been studied and modelled using VLBI observations by several authors (e.g. Walker et al. 2001; Gómez et al. 2001; Hardee et al. 2005). Our wavelet analyses reveal a timescale of 4.3 years at both 22 and $37 \mathrm{GHz}$. At $22 \mathrm{GHz}$ the timescale has been present for $85 \%$ of the total observing period lasting for 4.4 cycles. At $37 \mathrm{GHz}$ it has been present for $84 \%$ of the time and persisted for 4.6 cycles. In Paper I the DCF analyses showed a timescale of 4.2 years at $22 \mathrm{GHz}$ which is very close to what we obtained here. Both frequencies also show flare timescales of 1.4 years and in addition 0.5 years at $22 \mathrm{GHz}$ and 0.3 years at $37 \mathrm{GHz}$. These timescales are also seen in the DCF and SF analyses of Paper I. At $90 \mathrm{GHz}$ the timescale obtained is 2.7 years and it has been present for $73 \%$ of the time and continued for 4 cycles. This timescale is also very close to the DCF timescale of 2.9 years.

Visual inspection of the flux curve at $37 \mathrm{GHz}$ shows that indeed many of the larger flares have approximately 4.5 to 5 years between them but there are also additional flares in between. The last big flare included in our analysis at $37 \mathrm{GHz}$ peaked in the autumn of 2003. The next big flare was observed at the end of 2006 with an interval of 3 years between the flares. This indicates that the source is not strictly periodic but has shown a characteristic timescale of 4.3 years.

0736+017: This HPQ source was not detected by EGRET but has been listed as a possible $\gamma$-ray source to be detected with the new satellite missions AGILE and GLAST (Bach et al. 2007). In Metsähovi it has been monitored for over 20 years at 22 and $37 \mathrm{GHz}$ and for 11 years at $90 \mathrm{GHz}$. It exhibits rapid variability and in the optical domain it has been studied by several authors (e.g. Clements et al. 2003; Ramírez et al. 2004). The wavelet plot has a complex structure at all the frequency bands and we found multiple timescales for the source. The most significant and long-lasting ones are 2.7, 3.4 and 1.7 years at 22, 37 and $90 \mathrm{GHz}$, respectively. The timescales have repeated themselves 4 times at all the frequency bands and are also present for about half of the flux curve. The DCF analysis of Paper I shows very similar timescales of 2.8 years at 22 and $37 \mathrm{GHz}$ and 1.2 years at $90 \mathrm{GHz}$. At 22 and $37 \mathrm{GHz}$ there are multiple timescales present also in the DCF which confirms the complex variability of this source. Therefore it is also difficult to predict when the next flares could be expected.

OJ $248(0827+243)$ : This radio quasar was found by EGRET to be a bright $\gamma$-ray source. It has been monitored at Metsähovi for about 11 years both at 22 and $37 \mathrm{GHz}$. It has also been a target of multiwavelength campaigns (e.g. Bach et al. 2007) and has shown extremely fast apparent pattern velocity exceeding $25 c$ in the jet (Piner et al. 2006). The kiloparsec-scale jet is studied in more detail in Jorstad \& Marscher (2004). We found a timescale of 1.4 years at both frequency bands but it appears quite weak in strength and is classified as a weak timescale at $22 \mathrm{GHz}$. At $22 \mathrm{GHz}$ it has been present for $54 \%$ of the time and is discontinuous. It repeated 4.2 times during the time it has been present. At $37 \mathrm{GHz}$ the timescale has been present for $77 \%$ of the time and has a shortening trend changing the timescale from 1.4 to 1.1 years. The timescale repeated 6.3 times. In Paper I we measured timescales of 1.7 and 1.4 years at 22 and $37 \mathrm{GHz}$ respectively from the DCF analyses.

OJ $287(0851+202)$ : This BLO type object is one of the most studied AGNs. In Metsähovi it has been monitored for over 25 years at 22 and $37 \mathrm{GHz}$ and at $90 \mathrm{GHz}$ for 15 years. In the optical there is strong evidence of a period of about 12 years which is explained by several binary black hole models (e.g. Sillanpää et al. 1988; Lehto \& Valtonen 1996; Valtaoja et al. 2000; Valtonen et al. 2008). We can neither confirm nor disprove the existence of the same period at our frequency bands because the monitoring time is too short compared to the optical historical light curve which is over 100 years long. The source has also been studied with wavelets by Hughes et al. (1998). They used the continuous wavelet transform to study more than 20 years of data obtained in the University of Michigan Radio Observatory (UMRAO) at 4.8, 8 and $14.5 \mathrm{GHz}$. They found a persistent timescale of 1.66 years in the total flux and polarisation and another timescale of 1.12 years dominating the activity in the 1980s. They explained the modulations with a shock-in-jet model in which the permanent timescale is associated with the quiescent jet and the shorter timescale is due to the passage of a shock.

We found a timescale of 1.4 years at 22 and $90 \mathrm{GHz}$ and 1.7 years at $37 \mathrm{GHz}$. Both of these can be considered to be very similar to the ones obtained by Hughes et al. (1998), given the ambiguity in determining the exact time scale in our analysis. A very interesting phenomenon seen in the wavelet transform is that the timescale either weakened or totally disappeared between 1993 and 2000. Due to this the timescale is present only for $2 / 3$ of the time in all the frequency bands. It can be clearly seen in the flux density curve in Fig. 6 that the flux density level is generally lower during that time, even though smaller flares occur continuously. It is also possible to see in the wavelet plot that the number of rapid flares is lower during that period than in the more active states. Nevertheless the timescale repeated 10.2 times at $22 \mathrm{GHz}, 9.6$ times at $37 \mathrm{GHz}$ and 7.2 times at $90 \mathrm{GHz}$. All the timescales have a corresponding one in the DCF analysis of Paper I within 6 months of the one obtained from wavelet analysis. When studying the flare timescales we noticed timescales of 0.4 and 0.3 years at 22 and $37 \mathrm{GHz}$, respectively. These agree with timescales from the SF analysis in Paper I. It is difficult to associate the timescales with individual flares because the source exhibits continuous variability and is almost never in a quiescent state. 
OL 093 (1055+018): This HPQ source has been observed in Metsähovi for over 20 years at 22 and $37 \mathrm{GHz}$ and for almost 13 years at $90 \mathrm{GHz}$. The jet of this source is suggested to have a spine-sheath structure (Attridge et al. 1999; Pushkarev et al. 2005). We obtained a timescale of 4.3 years for the source at both of the 22 and $37 \mathrm{GHz}$ frequencies. The timescale repeated 4.4 times and is present for 85 and $80 \%$ of the flux curve at 22 and $37 \mathrm{GHz}$, respectively. Very similar timescales are measured in Paper I, where a DCF timescale of 4.7 and 4.4 years are obtained at 22 and $37 \mathrm{GHz}$, respectively. At $90 \mathrm{GHz}$ we obtain a timescale of 2.7 years but it repeated only 2.6 times. When examining the flux curve at $37 \mathrm{GHz}$ we see that indeed there are many flares with approximately $4-5$ years between them. The last flare included in the wavelet analysis peaked in 2002 and another large flare was observed in 2007, producing a time interval of 5 years between the flares. In addition there was a smaller flare peaking in 2004 which shows that even though the timescale of 4.3 years describes the larger events quite well, there is still more complex variability present in this source.

$4 C 38.41(1633+382)$ : This is another EGRET-detected $\gamma$-ray bright quasar which has been a target of many different observing programs (e.g. Katajainen et al. 2000; Jorstad et al. 2001; Lister \& Homan 2005; Bach et al. 2007). In Metsähovi it has been monitored for over 20 years at 22 and $37 \mathrm{GHz}$. It is also included in the wavelet study of Kelly et al. (2003) which used the UMRAO data at 4.8, 8 and $14.5 \mathrm{GHz}$. In their analysis no timescale was found for the source. We found a timescale of 3.4 years in both frequency bands but the wavelet plot has a complex structure and the variability timescale is weaker in the late 1980s and early 1990s. The timescale has been present for almost 3/4 of the flux curve in both frequency bands and repeated 4.8 and 5.1 times at the 22 and $37 \mathrm{GHz}$ frequencies, respectively. The timescale is not seen in DCF analysis of Paper I, probably because even though there are some flares with 3.5 years between them, it is not a timescale clearly seen to repeat in the flux curve.

PKS 1749+096: This BLO type object has been monitored in Metsähovi for 20 years at $22 \mathrm{GHz}$ and 25 years at $37 \mathrm{GHz}$. At $90 \mathrm{GHz}$ the data sets are 14 years long. The source has been a target of many Very Long Baseline observations (e.g. Homan et al. 2001; Wiik et al. 2001; Lister \& Homan 2005; Piner et al. 2007). We found a timescale of 2.7 years in all the frequency bands but the structure of the wavelet plot is very complex and the timescale is increasing in time from 1.7 to 2.7 years. At $90 \mathrm{GHz}$ it also repeated only 3.4 times while at $22 \mathrm{GHz} 4.4$ and at $37 \mathrm{GHz}$ 5 times. At all frequency bands the timescale has also been present for less than $70 \%$ of the time. Nevertheless timescales of 2.8 years at 22 and $37 \mathrm{GHz}$ and 2.3 years at $90 \mathrm{GHz}$ are also seen in the DCF analysis of Paper I. In addition we found flare timescales of 0.4 and 0.5 years at 22 and $37 \mathrm{GHz}$, respectively. Both are also seen within 0.2 years in the SF analysis of Paper I. The source exhibits rapid variability and has many large flares in the flux curve. Indeed many of the flares seem to be within 2.7 years of each other but there is also other activity present making the analysis complex.

\section{Discussion}

Our interest in using wavelets to study the timescales arose from the results of Paper I, which showed that many of the sources have changed their behaviour during the monitoring time and the timescales have changed over the years. A useful property of wavelets is that they show also when the timescale has been present and how it has changed. Many studies on individual sources have claimed the sources to be periodic or quasi-periodic based on results from Fourier-based methods. Our results show that only a very small number of sources actually show persistent timescales lasting over the total monitoring time (18 sources in which the timescale had lasted for over $90 \%$ of the total monitoring time). A good example is the source 1156+295 shown in Fig. 1. The wavelet timescale of 3.4 years is seen to be present only in the latter half of the flux curve. In Fig. 11 of Paper I the DCF and Lomb-Scargle periodogram analyses are shown for the same source. The DCF gives a timescale of 3.5 years and the Lomb-Scargle periodogram a timescale of 3.3 years which are very close to the timescale obtained with wavelets. With these methods, however, it was impossible to see that the source had changed its behaviour in the mid 1990s and the timescale was only present for the latter half of the monitoring period. In eight cases the timescale either disappeared or weakened at some point during the monitoring period and re-appeared later on. These changes cannot be detected by Fourier-based methods. Therefore we feel that wavelets should be used more when characteristic timescales and quasi-periodicities are studied.

In many cases the timescale also changed slowly over time. In 17 cases we saw a rising trend in the wavelet plot, which means a shortening timescale. These were often connected to long-lasting flares which showed shorter timescales towards the peak of the flare. This could indicate that there are more disturbances in the jet when a flare is growing and more shocks are developing in the jet, making the timescale shorter. Also, in six cases we detected a declining trend in the wavelet plot, indicating an increasing timescale. In these cases the activity is becoming less frequent and flares do not occur as often as earlier.

In Paper I we also found that the traditional Fourier-based methods have many problems when non-sinusoid flux curves are studied. In our sample this is the case for all sources. Especially with Lomb-Scargle periodogram, we obtain spurious timescales and other methods are needed to confirm the timescales. The DCF seemed slightly more reliable but it also has no means of showing if the timescale is discontinuous or changes in time. We compared our results from the wavelet analysis with those obtained in Paper I. The wavelet timescales are plotted against the DCF and periodogram timescales in Fig. 10. We can see a very good correspondence between the different timescales. We also calculated the Spearman rank correlation and between wavelet and DCF timescales we obtain a correlation of $r=0.58(p<$ $0.0001)$. Similarly between wavelet and periodogram timescales we obtain $r=0.55(p<0.0001)$. The correlation between the wavelet and periodogram timescales is affected by sources that have longer periodogram timescales of over 7 years but shorter wavelet timescales of 2 to 5 years. In almost all cases the periodogram analysis also revealed another timescale, similar to the wavelet timescale, but in our study we only used the most significant timescale from the periodogram analysis. Only for one source $(0736+017)$ did the periodogram analysis give only long timescales of over 8 years whereas the wavelet analysis gave short 2.7 and 3.4 years timescales. This source, however, has a complex structure in the wavelet plot (as seen in Fig. 4) and multiple timescales are seen.

The average timescales of wavelet and DCF analyses are also very similar with the difference being less than 6 months at all frequency bands. The averages of the periodogram analyses are slightly higher. The results show that both wavelet and DCF analyses give similar results and can be used to determine characteristic timescales. The advantage of wavelets is that we can also take into account how long a certain timescale has been present in the flux curve. 

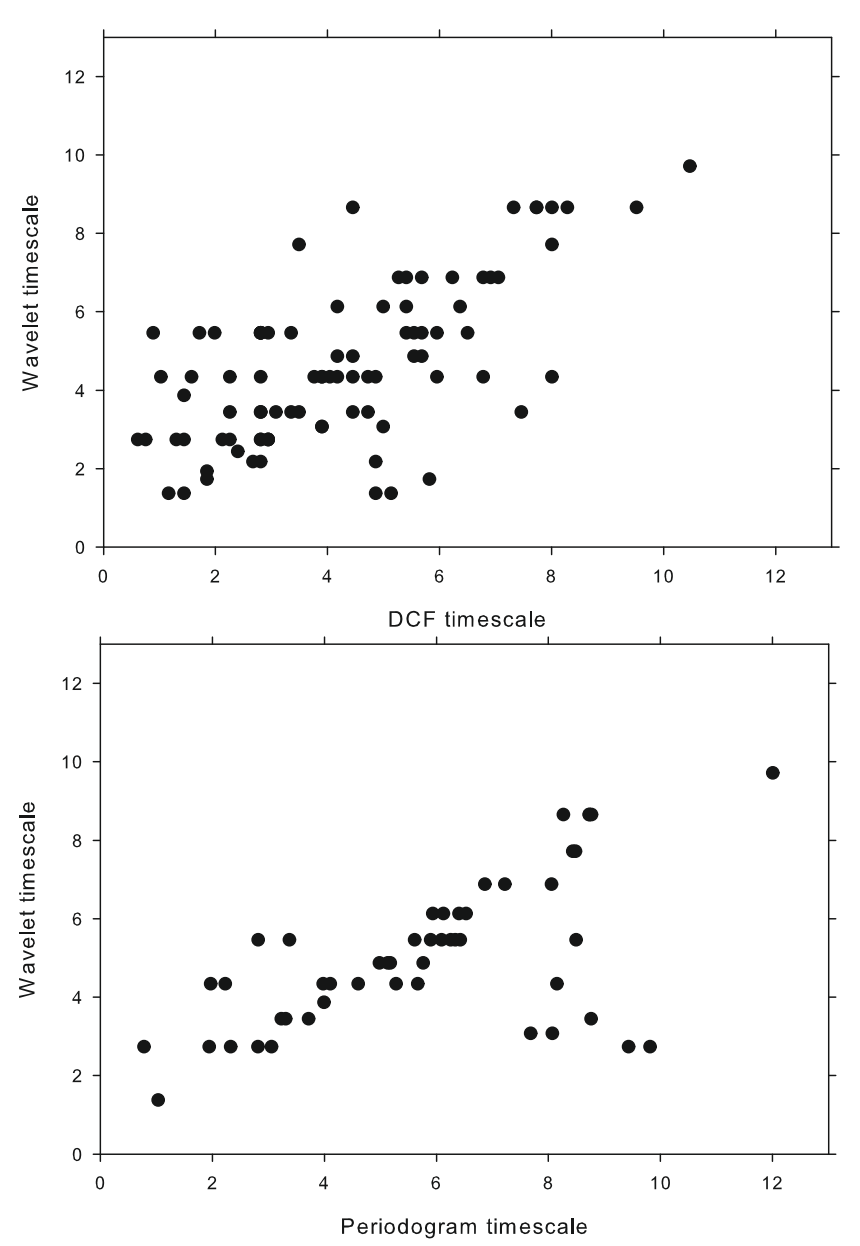

Fig. 10. Upper panel: long-term wavelet timescale against the DCF timescale from Paper I. Lower panel: the same wavelet timescale against the Lomb-Scargle periodogram timescale from Paper I.

In our sample we had nine sources for which Kelly et al. (2003) found variability timescales at some of the UMRAO frequencies. For one of those $(1823+568)$ we were not able to find any variability timescale in our analyses. In almost all cases we were able to find a similar timescale within 0.5 years of the timescale reported at one of the low frequencies. Only in one case was the timescale totally different $(1641+399)$ and in one case $(0212+735$ at $37 \mathrm{GHz})$ there was a difference of 0.7 years between the timescales. In three cases it was the timescale we refer to as the flare timescale that was similar to the lower frequency timescale. The similarity between the results from the two analyses supports use of the method.

Even though we found no strict periodicities in the radio behaviour of any of our sources, either in Paper I or in this paper, we wanted to investigate the possibility of "predicting" upcoming active states in these sources. Many of these sources have changed their behaviour during the 20 years of observations (for example 1156+295 in Fig. 1), thus such predictions would be based purely on their statistical behaviour. This attempt was partly motivated by the multifrequency support observations we participate in: it would be immensely useful to be able to prioritise those sources that are likely to enter an active state fairly soon. In particular, we were interested to see if we can predict which sources would be likely to flare during the early operation of the upcoming gamma-ray satellite GLAST. Our earlier work (e.g. Tornikoski et al. 2002; Lähteenmäki \& Valtaoja 2003) has shown that strong gamma-ray activity usually corresponds to a growing flare in the radio regime. Therefore sources that are active in the radio domain are potential sources to be detected by GLAST.

Thus, we wanted to see which sources in our sample would be good candidates for strong flaring within the timeframe 20082009 , excluding a set of sources that have almost continuous and complex variability at $37 \mathrm{GHz}$, such as OJ 287 and BL Lac. From the wavelet timescales at $37 \mathrm{GHz}$, combined with the timescale analysis from Paper I and with visual examination of light curves until the end of 2007, we came up with a list of six sources that according to the wavelet analysis and at least one other method used in Paper I show semi-periodicity with such timescales that a strong radio flare could be anticipated to occur within 2008-2009. The sources include one GAL type object $(0007+106)$, two HPQs $(0234+285,1156+295)$, two LPQs $(0333+321,2145+067)$ and one BLO $(1749+096)$.

\section{Conclusions}

We studied a sample of 80 sources with the continuous wavelet transform using data at 22, 37 and $90 \mathrm{GHz}$. Our aim was to study the variability behaviour of the sources and also to better understand the method and to compare it with Fourier-based methods. We found no clear periodicities in the sources. Instead in most of the sources the timescales appeared only for a short time in the total monitoring period or changed over the years. These kind of properties are not revealed with Fourier-based methods and therefore wavelets should be used more when quasi-periodicities are studied. In our sample only $1 / 4$ had a timescale that had been present for at least 4 cycles. In eight of these sources the timescale was present in more than one frequency band. This shows that the sources are never strictly periodic and conclusions about periodicities in the radio frequencies should be drawn with caution.

The average timescale from the wavelet analysis is 4.5 years which is the same as was obtained in the DCF analysis of Paper I. This timescale should not be affected by the length of the monitoring period because we used all the sources in which the timescale had repeated at least twice in the analysis and therefore longer timescales also are included in the calculations. The weakness of wavelets is that we cannot detect the timescale with the same accuracy as with DCF or periodograms and therefore both type of methods should be used when periodicities or quasiperiodicities are searched for. The wavelets should be used to verify whether the timescale is persistent or short-lived. If a persistent timescale is found, the Fourier methods can be used to accurately determine the timescale. In our analysis where the characteristic timescales are studied, the accuracy of wavelets is enough to determine the approximate timescale.

Acknowledgements. We acknowledge the support of the Academy of Finland (project numbers 212656 and 205793).

\section{References}

Attridge, J. M., Roberts, D. H., \& Wardle, J. F. C. 1999, ApJ, 518, L87

Bach, U., Raiteri, C. M., Villata, M., et al. 2007, A\&A, 464, 175

Ciprini, S., Takalo, L., Tosti, G., et al. 2007, A\&A, 467, 465

Clements, S. D., Jenks, A., \& Torres, Y. 2003, AJ, 126, 37

Foster, G. 1996, AJ, 112, 1709

Gómez, J.-L., Marscher, A. P., Alberdi, A., Jorstad, S. G., \& Agudo, I. 2001, ApJ, 561, L161

Hardee, P. E., Walker, R. C., \& Gómez, J. L. 2005, ApJ, 620, 646 
Homan, D. C., Ojha, R., Wardle, J. F. C., et al. 2001, ApJ, 549, 840 Hovatta, T., Tornikoski, M., Lainela, M., et al. 2007, A\&A, 469, 899 (Paper I) Hughes, P. A., Aller, H. D., \& Aller, M. F. 1998, ApJ, 503, 662

Jorstad, S. G., \& Marscher, A. P. 2004, ApJ, 614, 615

Jorstad, S. G., Marscher, A. P., Mattox, J. R., et al. 2001, ApJS, 134, 181

Kadler, M., Hughes, P. A., Ros, E., Aller, M. F., \& Aller, H. D. 2006, A\&A, 456, L1

Katajainen, S., Takalo, L. O., Sillanpää, A., et al. 2000, A\&AS, 143, 357

Kellermann, K. I., Lister, M. L., Homan, D. C., et al. 2004, ApJ, 609, 539

Kelly, B. C., Hughes, P. A., Aller, H. D., \& Aller, M. F. 2003, ApJ, 591, 695

Lähteenmäki, A., \& Valtaoja, E. 2003, ApJ, 590, 95

Lehto, H. J. 1999, in BL Lac Phenomenon, ed. L. O. Takalo, \& A. Sillanpää (San Francisco: ASP), ASP Conf. Ser., 159, 257

Lehto, H. J., \& Valtonen, M. J. 1996, ApJ, 460, 207

Lister, M. L. 2001, ApJ, 562, 208

Lister, M. L., \& Homan, D. C. 2005, AJ, 130, 1389

Nieppola, E., Tornikoski, M., Lähteenmäki, A., et al. 2007, AJ, 133, 1947

Piner, B. G., Bhattarai, D., Edwards, P. G., \& Jones, D. L. 2006, ApJ, 640, 196

Piner, B. G., Mahmud, M., Fey, A. L., \& Gospodinova, K. 2007, AJ, 133, 2357

Priestley, M. B. 1997, in Statistical Challenges in Modern Astronomy II, ed. G. J. Babu, \& E. D. Feigelson, 283

Pushkarev, A. B., Gabuzda, D. C., Vetukhnovskaya, Y. N., \& Yakimov, V. E. 2005, MNRAS, 356, 859
Ramírez, A., de Diego, J. A., Dultzin-Hacyan, D., \& González-Pérez, J. N. 2004, A\&A, 421, 83

Reuter, H.-P., Kramer, C., Sievers, A., et al. 1997, A\&AS, 122, 271

Salonen, E., Teräsranta, H., Urpo, S., et al. 1987, A\&AS, 70, 409

Scargle, J. D. 1997, in Statistical Challenges in Modern Astronomy II, ed. G. J. Babu, \& E. D. Feigelson, 333

Sillanpää, A., Haarala, S., \& Valtonen, M. J. 1988, ApJ, 325, 628

Steppe, H., Salter, C. J., Chini, R., et al. 1988, A\&AS, 71, 317

Steppe, H., Liechti, S., Mauersberger, R., et al. 1992, A\&AS, 96, 441

Steppe, H., Paubert, G., Sievers, A., et al. 1993, A\&AS, 102, 611

Teräsranta, H., Tornikoski, M., Valtaoja, E., et al. 1992, A\&AS, 94, 121

Teräsranta, H., Tornikoski, M., Mujunen, A., et al. 1998, A\&AS, 132, 305

Teräsranta, H., Achren, J., Hanski, M., et al. 2004, A\&A, 427, 769

Teräsranta, H., Wiren, S., Koivisto, P., Saarinen, V., \& Hovatta, T. 2005, A\&A, 440, 409

Tornikoski, M., Valtaoja, E., Teräsranta, H., et al. 1996, A\&AS, 116, 157

Tornikoski, M., Lähteenmäki, A., Lainela, M., \& Valtaoja, E. 2002, ApJ, 579, 136

Valtaoja, E., Teräsranta, H., Tornikoski, M., et al. 2000, ApJ, 531, 744

Valtonen, M. J., Lehto, H. J., Nilsson, K., et al. 2008, Nature, 452, 851

Walker, R. C., Benson, J. M., Unwin, S. C., et al. 2001, ApJ, 556, 756

Wiik, K., Valtaoja, E., \& Leppänen, K. 2001, A\&A, 380, 72 
T. Hovatta et al.: Wavelet analysis of a large sample of AGN at high radio frequencies, Online Material $p 1$

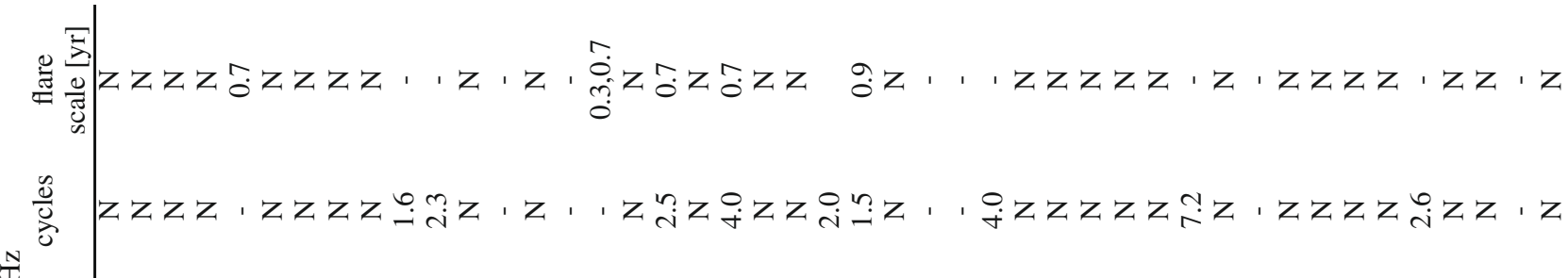

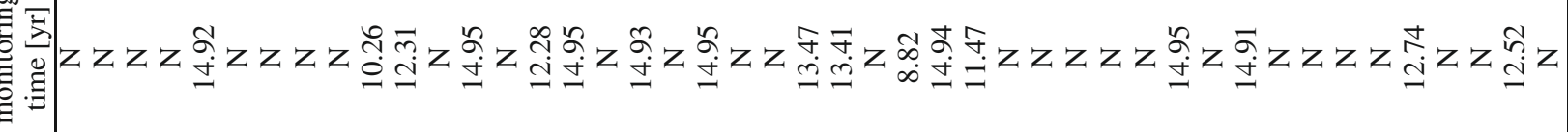

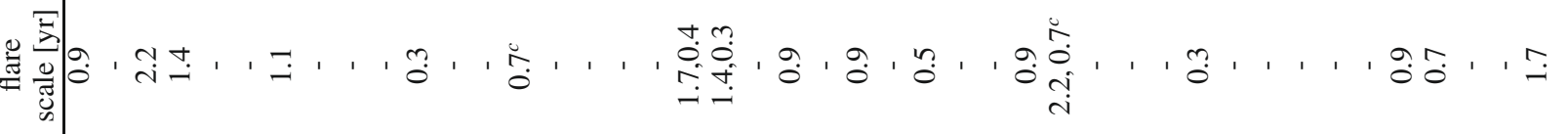

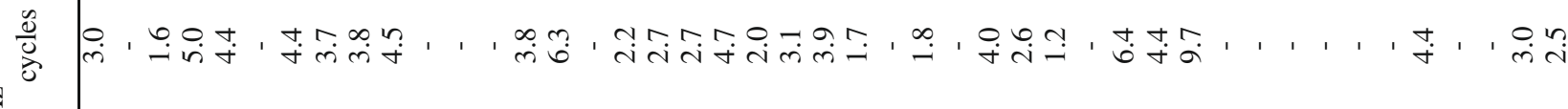
夌

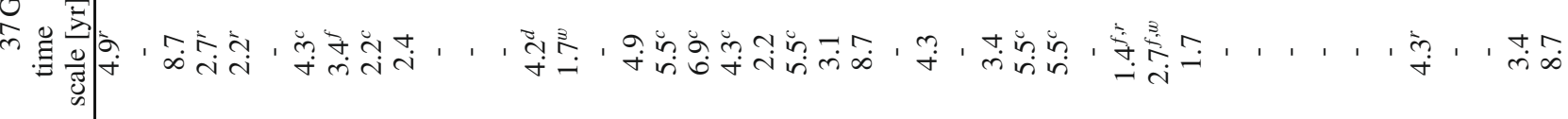

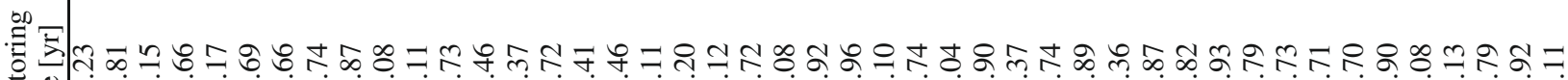

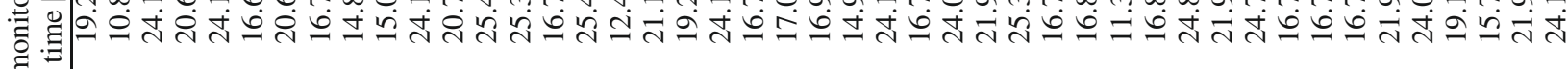

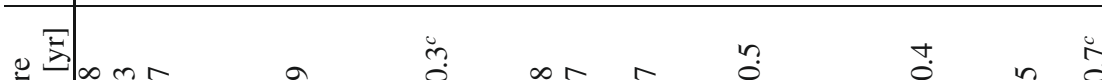

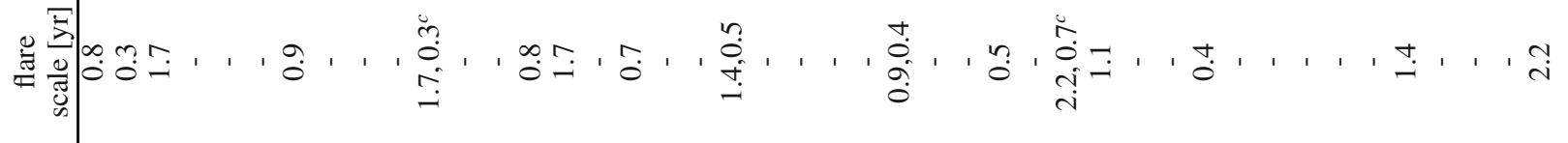

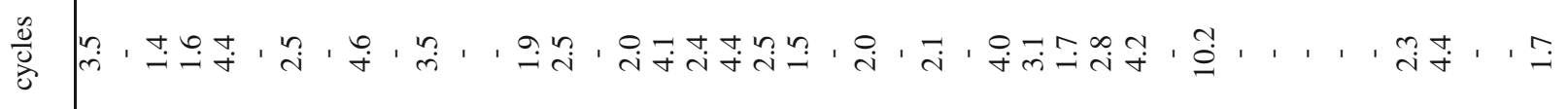
소

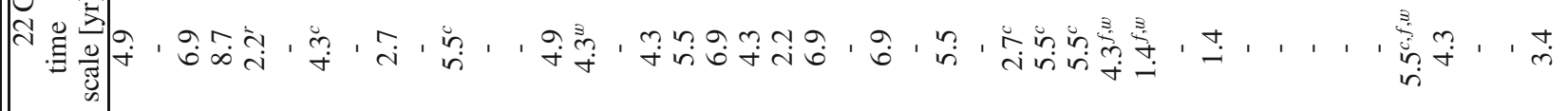

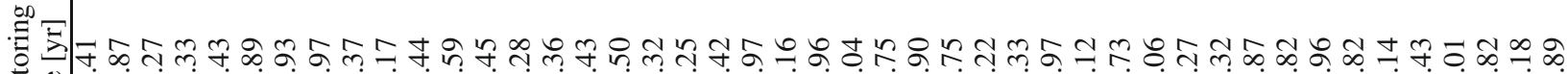
药

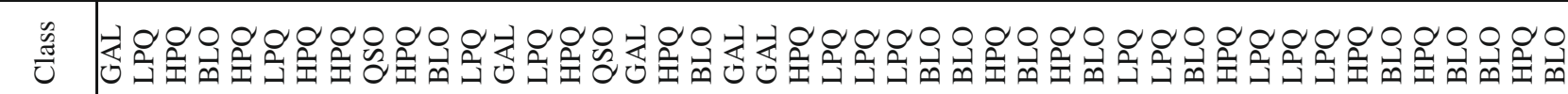

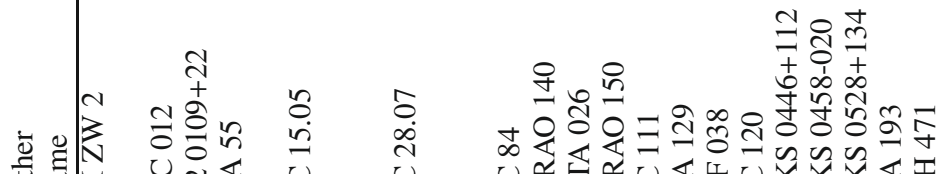

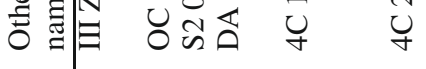

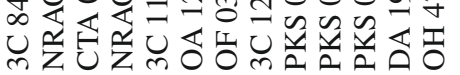

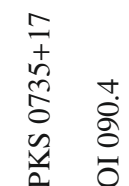

苛 Nữư

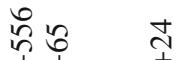

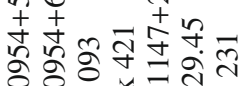

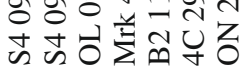

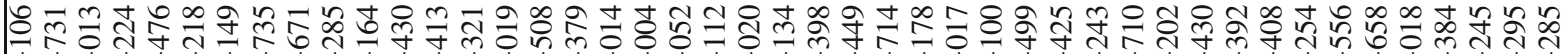

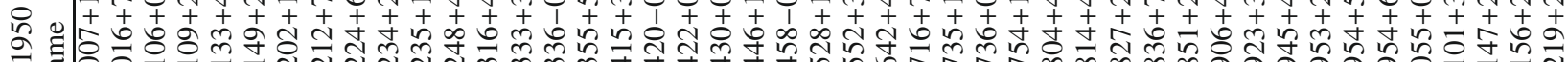

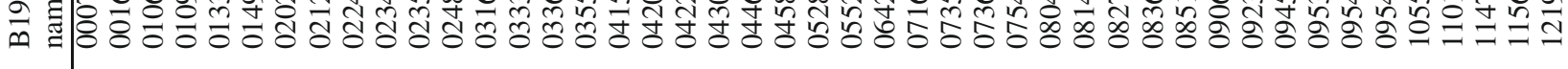


T. Hovatta et al.: Wavelet analysis of a large sample of AGN at high radio frequencies, Online Material p 2

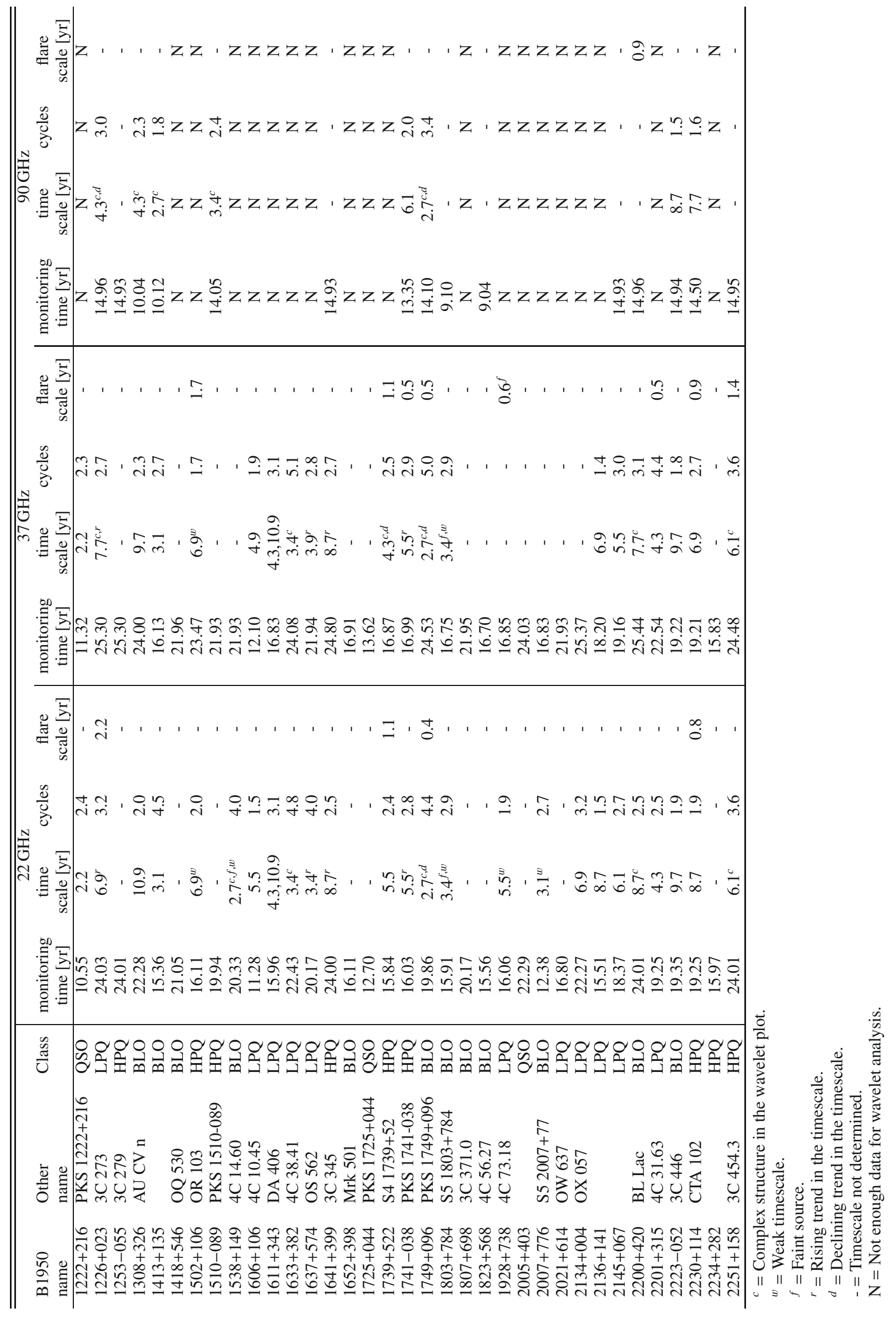


T. Hovatta et al.: Wavelet analysis of a large sample of AGN at high radio frequencies, Online Material p 3

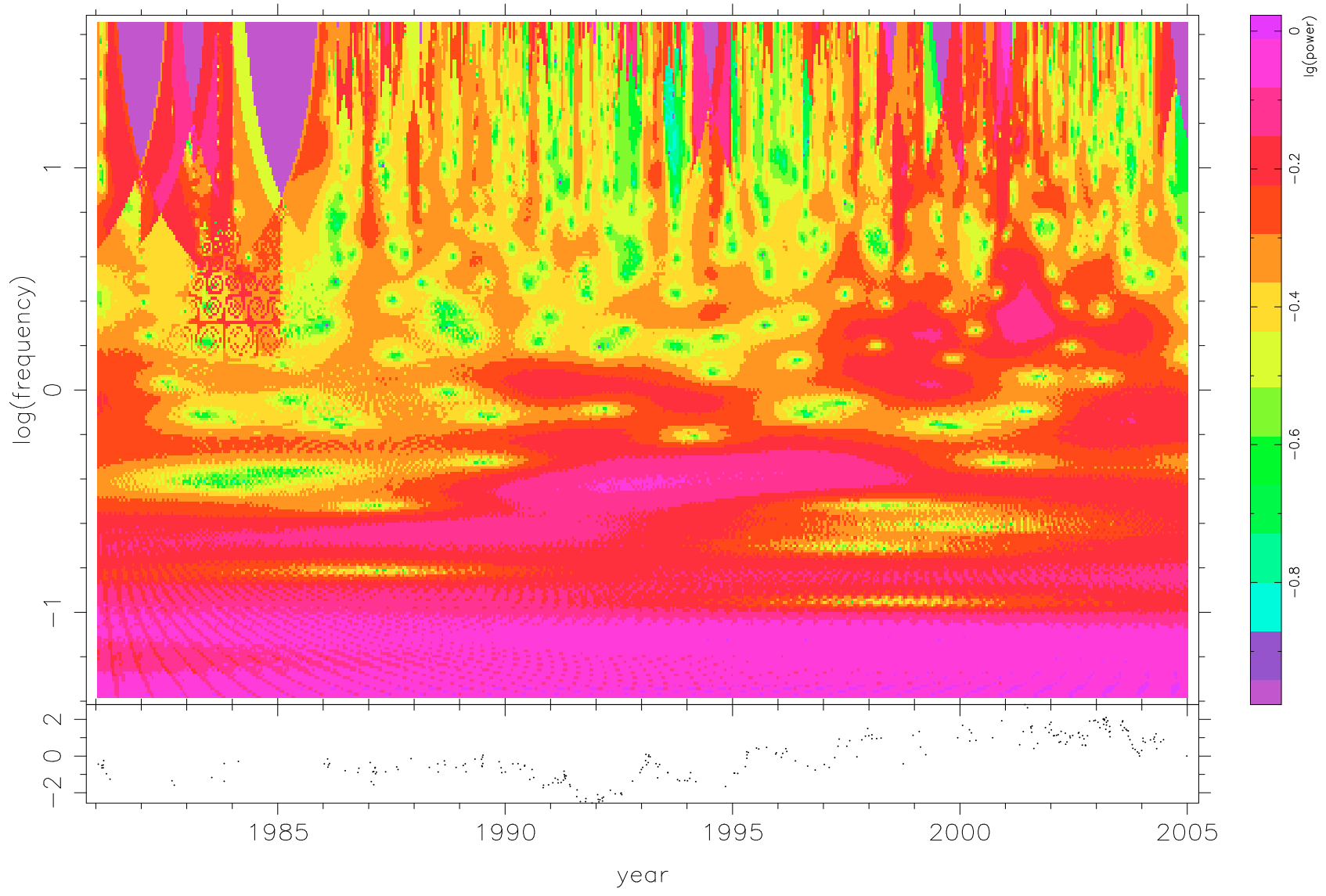

Fig. 2. Wavelet transform of the source DA $55(0133+476)$ at $37 \mathrm{GHz}$.

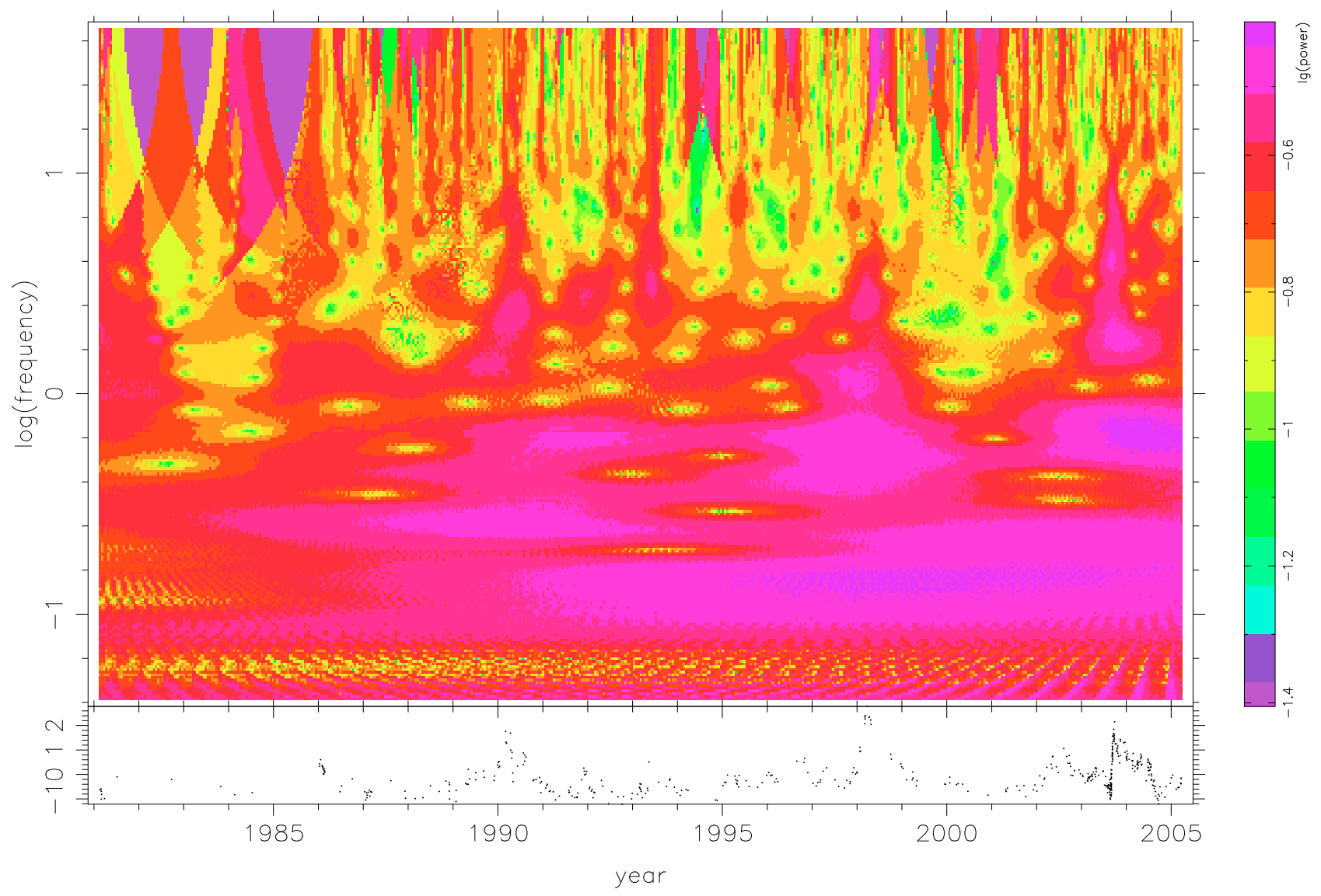

Fig. 3. Wavelet transform of the source 3C $120(0430+052)$ at $37 \mathrm{GHz}$. 
T. Hovatta et al.: Wavelet analysis of a large sample of AGN at high radio frequencies, Online Material p 4

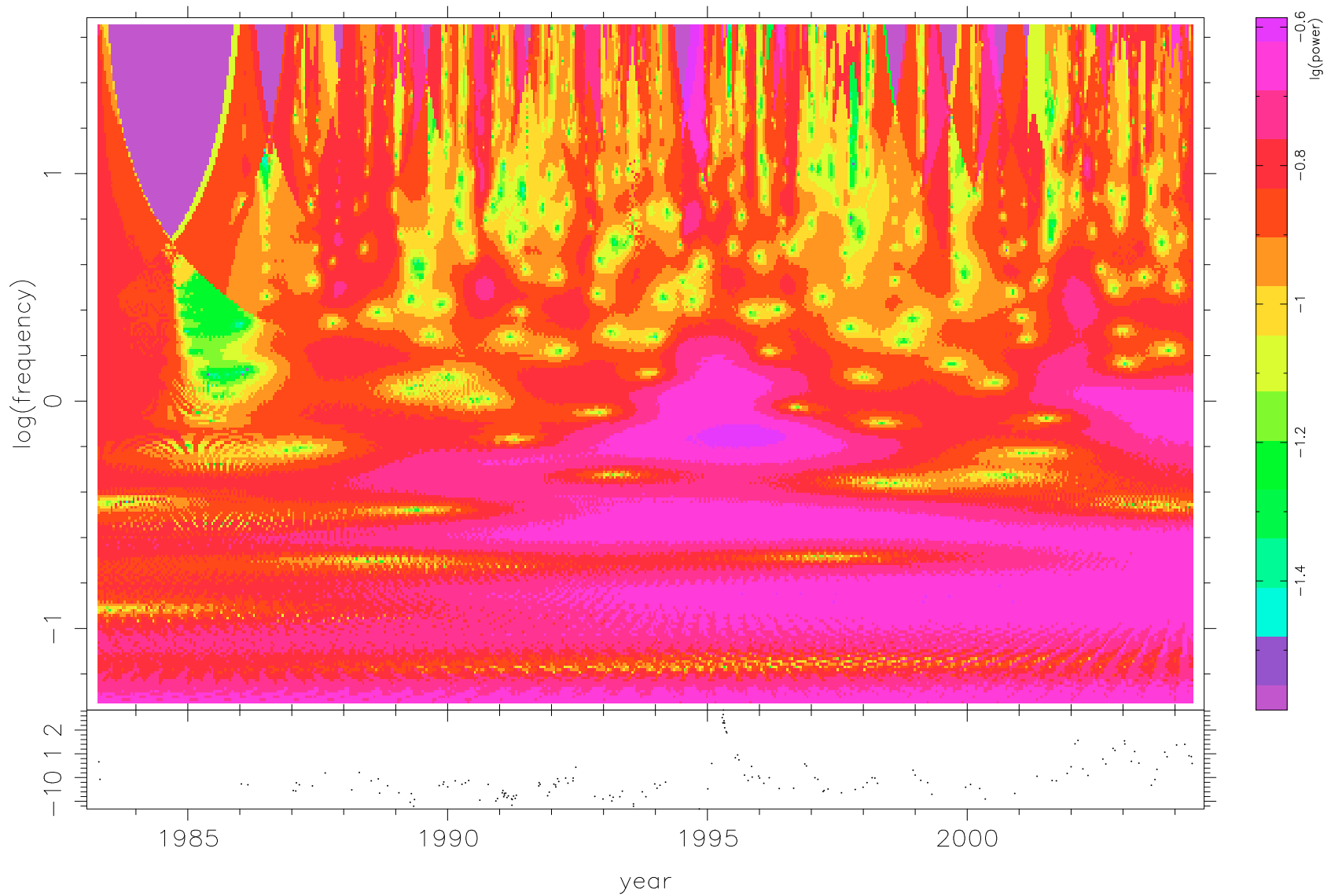

Fig. 4. Wavelet transform of the source $0736+017$ at $37 \mathrm{GHz}$.

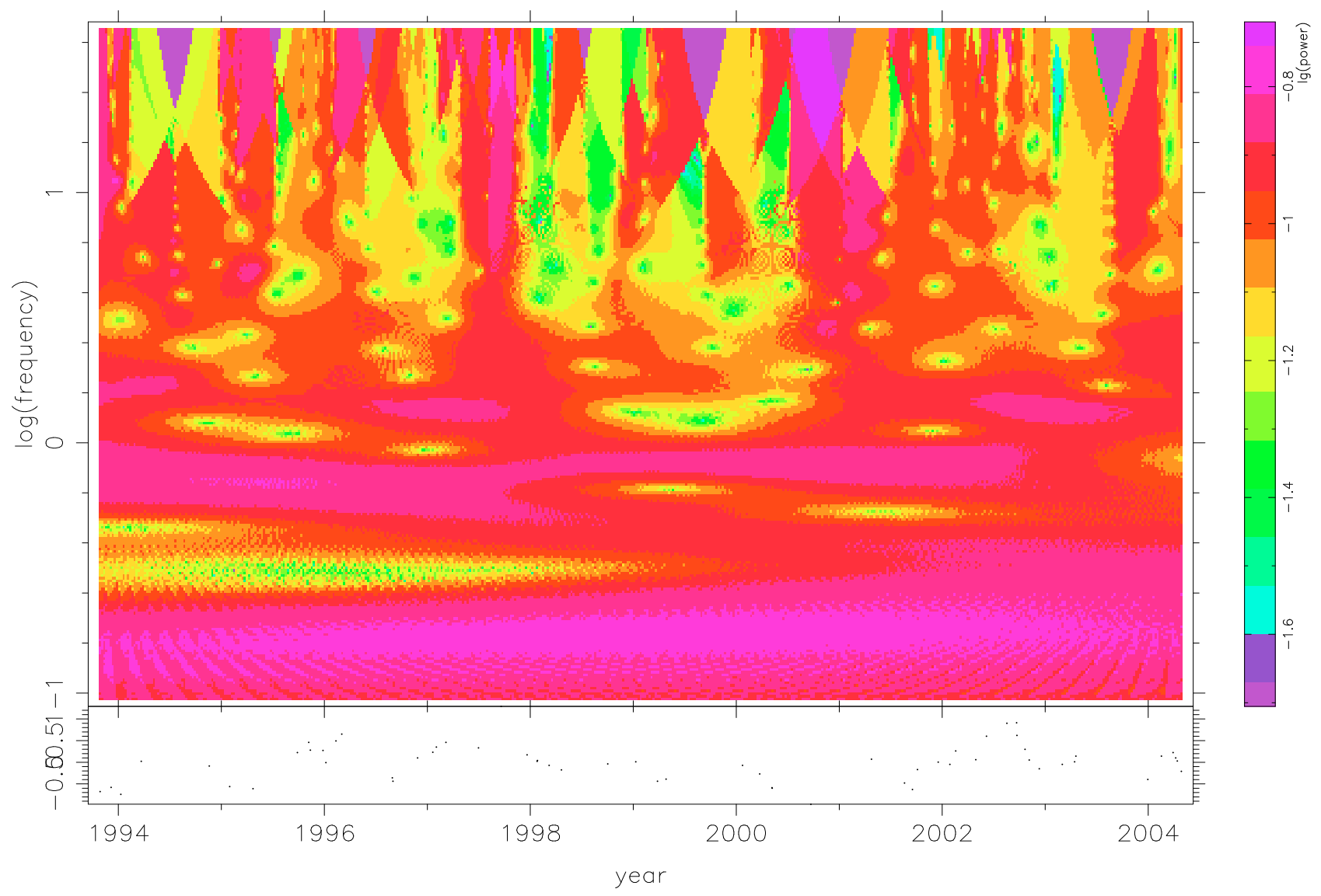

Fig. 5. Wavelet transform of the source OJ $248(0827+243)$ at $37 \mathrm{GHz}$. 
T. Hovatta et al.: Wavelet analysis of a large sample of AGN at high radio frequencies, Online Material p 5

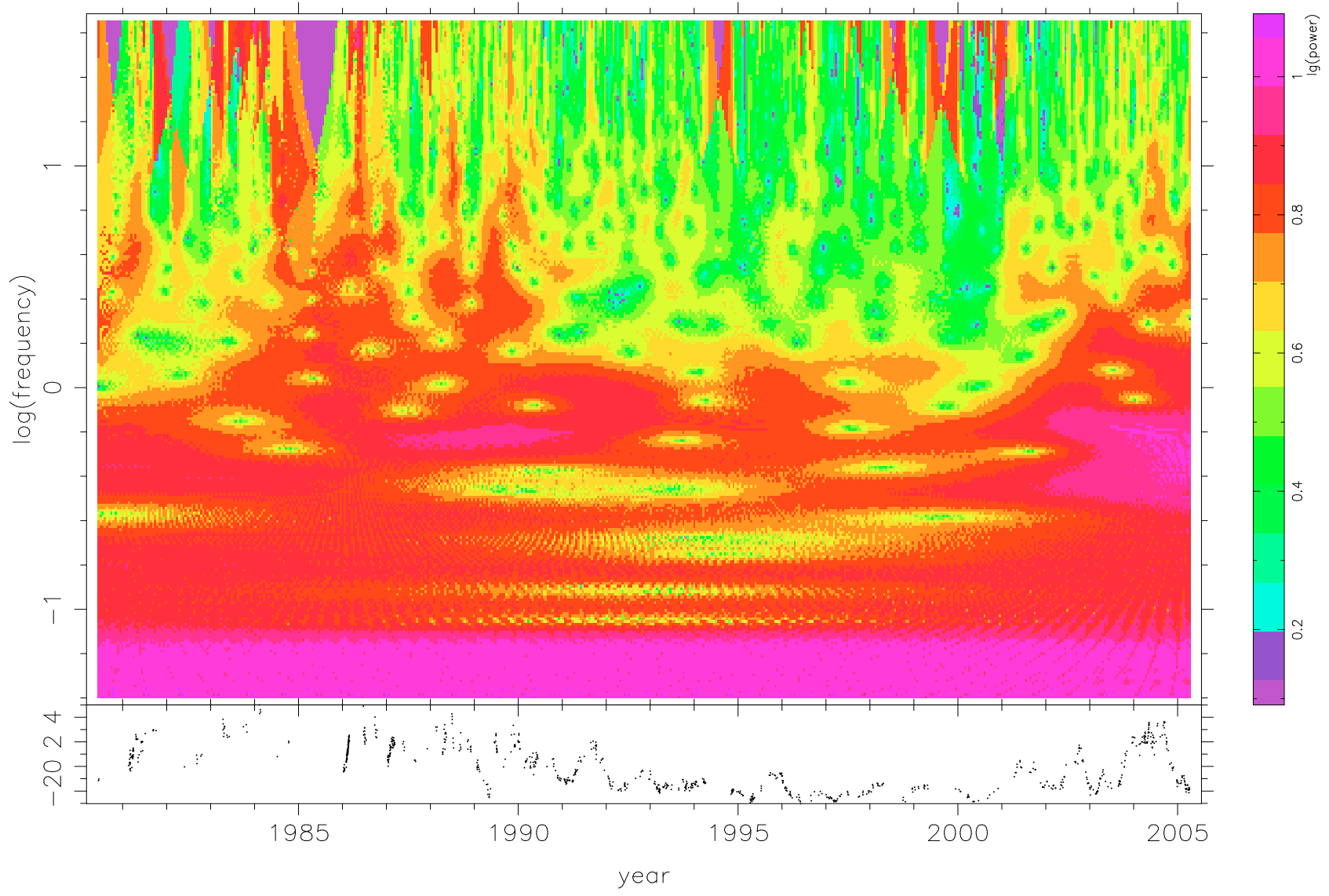

Fig. 6. Wavelet transform of the source OJ $287(0851+202)$ at $37 \mathrm{GHz}$.

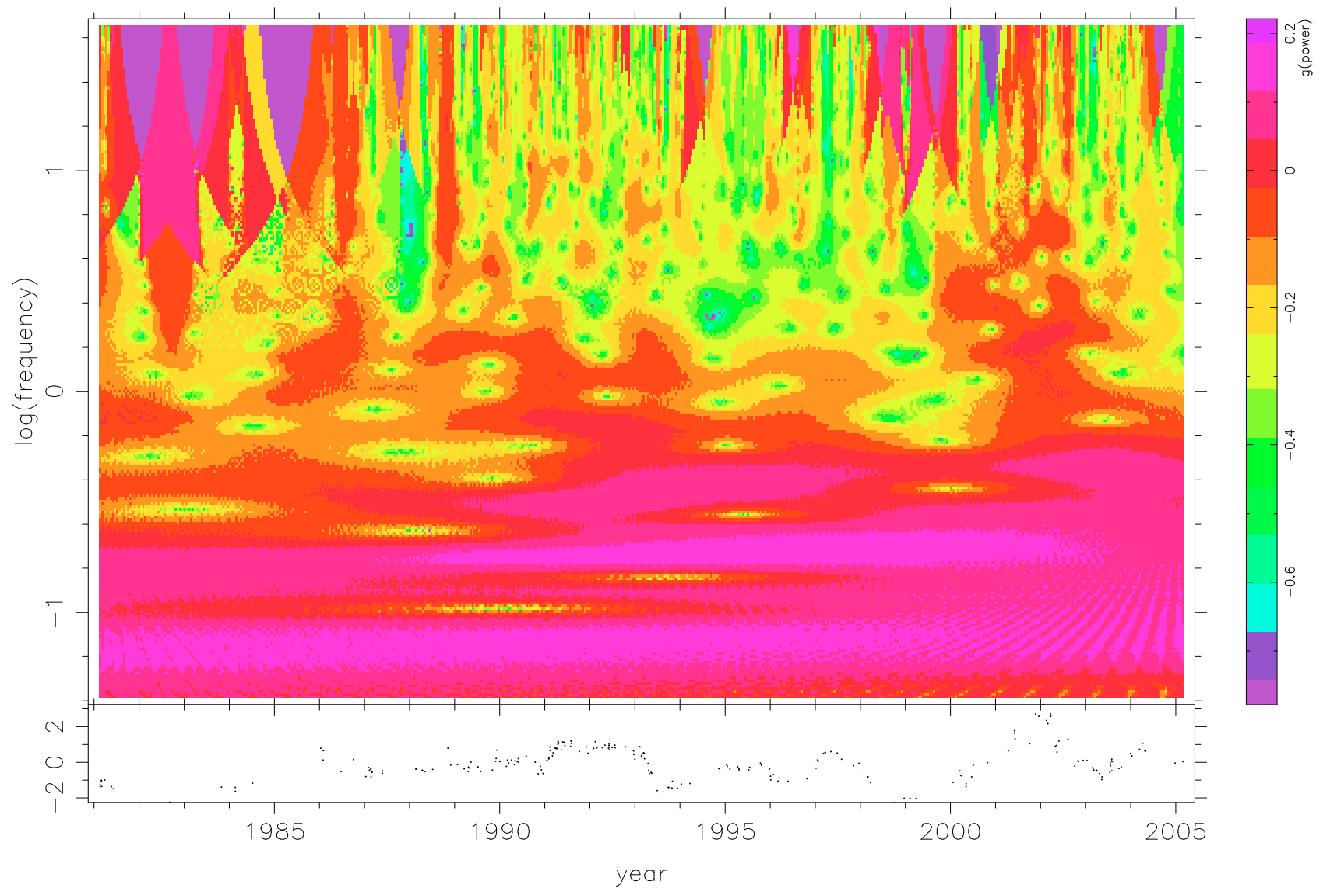

Fig. 7. Wavelet transform of the source OL $093(1055+018)$ at $37 \mathrm{GHz}$. 
T. Hovatta et al.: Wavelet analysis of a large sample of AGN at high radio frequencies, Online Material p 6

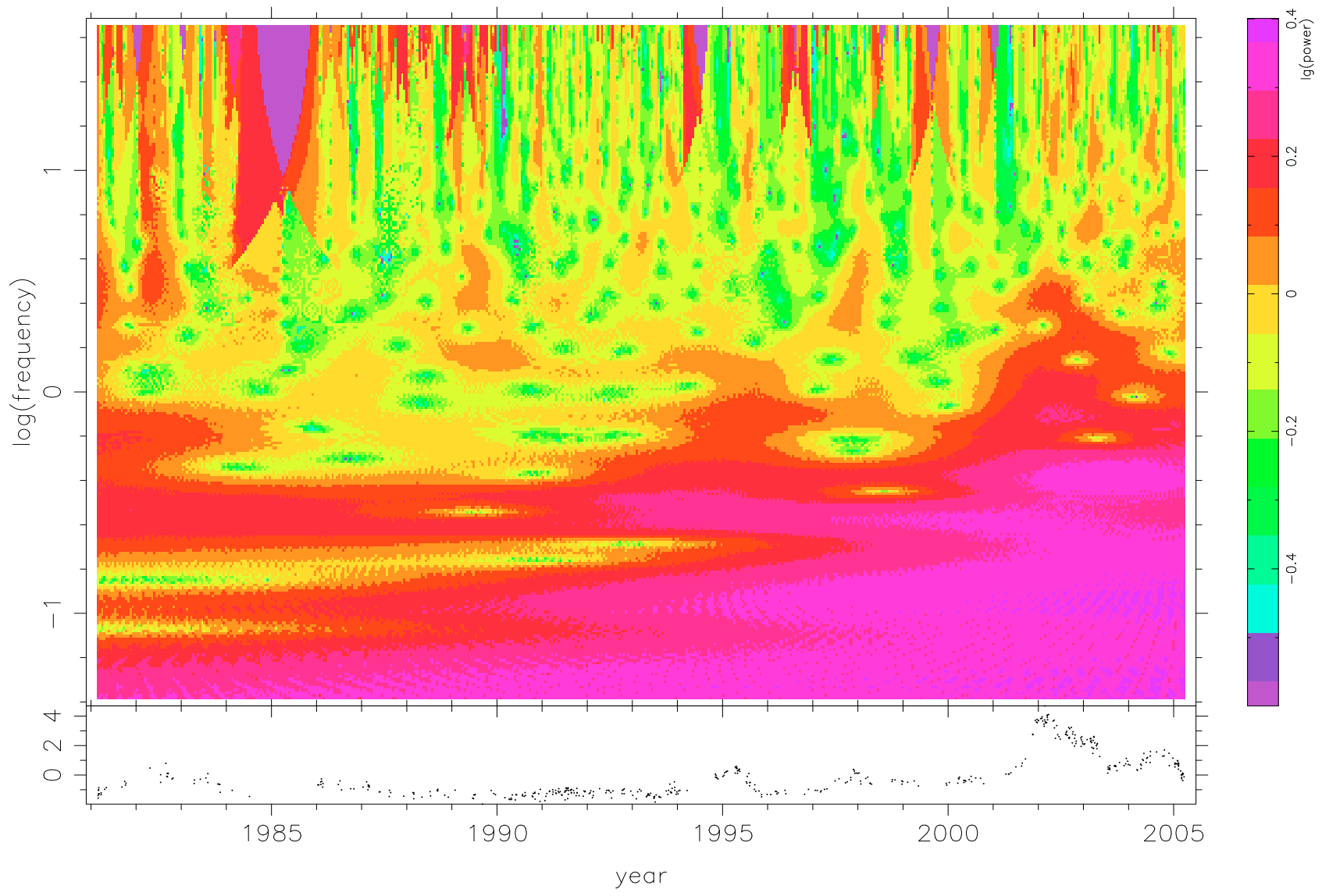

Fig. 8. Wavelet transform of the source 4C $38.41(1633+382)$ at $37 \mathrm{GHz}$.

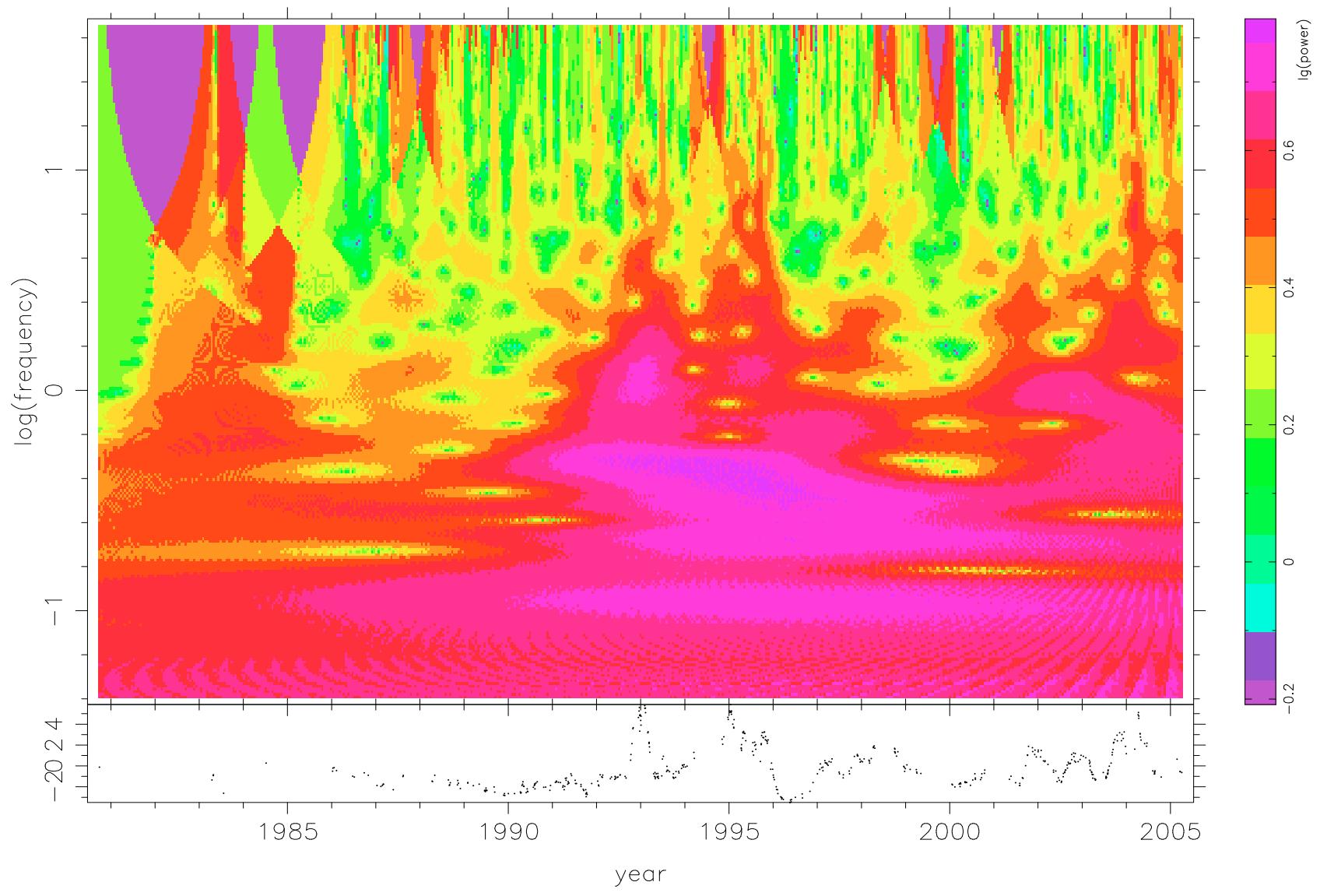

Fig. 9. Wavelet transform of the source PKS $1749+096$ at $37 \mathrm{GHz}$. 